H. Tanigawa

Nagoya Math. J.

Vol. 127 (1992), 117-128

\title{
HOLOMORPHIC FAMILIES OF GEODESIC DISCS IN INFINITE DIMENSIONAL TEICHMÜLLER SPACES
}

\author{
HARUMI TANIGAWA
}

\section{Introduction}

The theory of quasiconformal mappings plays an important role in Teichmüller theory. The Teichmüller spaces of Riemann surfaces are defined as quotient spaces of the spaces of Beltrami differentials, and the Teichmüller distances are defined to measure quasiconformal deformations between the Riemann surfaces representing points in the Teichmüller spaces. The Teichmüller spaces are complex Banach manifolds equipped with natural complex structures such that the canonical projections are holomorphic. It is known (see Gardinar [4]) that the Teichmüller distance, defined independently of the complex structures, coincides with the Kobayashi distance.

In spite of the naturality of the definition of a Teichmüler space as a quotient of Beltrami differentials, for given two Beltrami differentials it is hard to determine whether they are equivalent or not. For this reason, it is not trivial to describe geodesic lines with respect to the Teichmüller-Kobayashi metric.

In finite dimensional cases, each pair of points determines a unique geodesic line through them, and the geodesic line is obtained by the unique extremal differential called a Teichmüller differential. Namely, each geodesic line through the base point is represented as $\{t \mu:-1<t<1\}$ with a Teichmüller differential $\mu$. On the other hand, little was known about infinite dimensional cases for a long time. In this case, it is known that there exists a point $p$ which contains two different extremal Beltrami differentials $\mu_{1}$ and $\mu_{2}$, and both of the sets $\left\{t \mu_{1} /\right.$ $\left.\left\|\mu_{1}\right\|_{\infty}:-1<t<1\right\}$ and $\left\{t \mu_{2}\left\|\mu_{2}\right\|_{\infty}:-1<t<1\right\}$ are geodesic lines. However, one cannot conclude immediately that these lines are distinct, since equivalence of $\mu_{1}$ and $\mu_{2}$ does not imply equivalence or non-equivalence of $t \mu_{1}$ and $t \mu_{2}$ for $t \neq 1$.

Recently, L. Zhong [20] showed non-uniqueness of geodesic lines through

Received October 17, 1991. 
certain points in the universal Teichmüller space. In this paper, we shall construct, in any infinite dimensional Teichmüller space, a non-trivial family with a complex analytic parameter, of geodesic discs through a pair of points. Our proof is not a direct extension of [20] but makes full use of the characteristic properties of ends with infinite hyperbolic area, pointed out by Taniguchi [16] and Maitani [10]. Our construction is related to the following problem.

For a finite dimensional Teichmüller space $T(S)$, we used the equality of the Kobayashi distances and the Teichmüller distances, together with properties of the boundary $\partial T(S)$ (in the sense of Bers), to show certain rigidity property of proper holomorphic mappings of $\Delta$ into $T(S)$, where $\Delta$ stands for the unit disc $\{\boldsymbol{z} \in \mathbf{C} ;|z|<1\}$ ([17]). Namely, we showed that no proper holomorphic mapping can be deformed with a complex analytic parameter. Here, we have a natural question : is the same true for proper holomorphic mappings into an infinite dimensional Teichmüller space $T(R)$ ? To this question, Professor C. McMullen suggesed how to construct non-rigid holomorphic proper mappings of $\Delta$ into infinite dimensional Teichmüller spaces (oral communication). His idea is as follows (see Sections 2 and 3 for details): on an analytically infinite Riemann surface $R$ there exists an extremal Beltrami differential $\mu$ with a degenerate Hamilton sequence (cf. Section 2). Replacing $\mu$ by 0 on a local disc, we have an extremal Beltrami differential on $R$ which vanishes on the local disc, in contrast with the case of an analytically finite Riemann surface. Deforming such a Beltrami differential on the local disc, we get a non-trivial family of holomorphic proper mappings of $\Delta$ into $T(R)$ with a complex analytic parameter. We develop his idea to construct a holomorphic family of geodesic discs. Of course, a holomorphic isometry $\Delta \rightarrow T(R)$ in such a family is a non-rigid proper holomorphic mapping.

In the last section, we shall show that there still exist rigid holomorphic mappings into infinite dimensional Teichmüller spaces. We investigate the boundary behavior of a holomorphic mapping of $\Delta$ into $T(R)$ and give a sufficient condition for holomorphic mappings into $T(R)$ to be rigid.

The author would like to thank Professor C. McMullen and Professor H. Shiga for useful suggestion and information.

\section{Notation}

In this section we fix our notation and recall some known facts which we use. See Lehto [9] for details.

Let $R$ be a Riemann surface with a hyperbolic metric, and let $p: \mathbf{H} \rightarrow R$ be a universal covering map with a covering transformation group $\Gamma$, where $\mathbf{H}$ denotes 
the upper half plane.

Denote by $B_{1}(R)$ the space of all Beltrami differentials on $R$ with supremum norms smaller than 1 . Each element $\tau \in B_{1}(R)$ lifts to a $(-1,1)$-form $\tilde{\tau}=p^{*} \tau$ for $\Gamma$ on $\mathbf{H}$, namely, $\tilde{\tau} \circ \gamma \times \overline{\gamma^{\prime}} / \gamma^{\prime}=\tilde{\tau}$ for every $\gamma \in \Gamma$. lt is well-known that there exists unique quasiconformal homeomorphism $f^{\tilde{\tau}}: \mathbf{H} \rightarrow \mathbf{H}$ with Beltrami differen tial $\tilde{\tau}$ whose extension to $\mathbf{H} \cup \mathbf{R}$ fixes 0,1 and $\infty$, where $\mathbf{R}$ stands for the real axis. This quasiconformal homeomorphism $f^{\tilde{\tau}}$ induces a group isomorphism of the Fuchsian group $\Gamma$ onto the Fuchsian group $f^{\tilde{\tau}} \Gamma\left(f^{\tilde{\tau}}\right)^{-1}$. The Fuchsian group $f^{\tilde{\tau}} \Gamma\left(f^{\tilde{\tau}}\right)^{-1}$ determines a Riemann surface $R^{\tau}=\mathbf{H} / f^{\tilde{\tau}} \Gamma\left(f^{\tilde{\tau}}\right)^{-1}$ and $f^{\tilde{\tau}}$ is projected to a quasiconformal mapping $f^{\tau}: R \rightarrow R^{\tau}$. Thus each element $\tau \in B_{1}(R)$ determines a pair of a Riemann surface $R^{\tau}$ and a quasiconformal mapping $f^{\tau}: R \rightarrow R^{\tau}$ with Beltrami differential $\tau$. (In general, a Beltrami differential on $R$ determines a quasiconformal mapping uniquely up to left compositions of conformal mappings. We have defined $f^{\tau}$ as above in order to cancel this ambiguity for arguments in following sections.) Two elements $\tau_{1}$ and $\tau_{2}$ in $B_{1}(R)$ are said to be equivalent provided $f^{\tilde{\tau}_{1}}\left|\mathbf{R} \equiv f^{\tau_{2}}\right| \mathbf{R}$. It is known that this equality holds if and only if $R^{\tilde{\tau}_{1}}$ $=R^{\tilde{\tau}_{2}}$ and $f^{\tilde{\tau}_{2}} \circ\left(f^{\tilde{\tau}_{1}}\right)^{-1}$ is homotopic to the identity with a homotopy constant on the border of $R$. An element $\tau \in B_{1}(R)$ is said to be trivial if it is equivalent to 0 . A quasiconformal mapping with a trivial differential is also said to be trivial.

The Teichmuller space $T(R)$ of $R$ is the quotient space of $B_{1}(R)$ by the above equivalence relation. For each $\tau \in B_{1}(R)$, let $[\tau]$ denote the point of $T(R)$ deter mined by $\tau$. It is known that $T(R)$ is a Banach manifold equipped with a natural complex structure such that the canonical projection $\pi: B_{1}(R) \rightarrow T(R)$ is holomorphic.

Let $A(R)$ denote the space of all holomorphic integrable quadratic differentials on $R$. The space $A(R)$ is identified with the cotangent space of $T(R)$ at [0]. The dimension of $T(R)$ and that of $A(R)$ are finite if and only if $R$ is analytically finite, or equivalently, if and only if $\Gamma$ is a finitely generated Fuchsian group of the first kind.

The Teichmüller distance $d\left(\left[\mu_{0}\right],\left[\nu_{0}\right]\right)$ of two points $\left[\mu_{0}\right]$ and $\left[\nu_{0}\right]$ in $T(R)$ is defined by

$$
d\left(\left[\mu_{0}\right],\left[\nu_{0}\right]\right)=\frac{1}{2} \inf \left\{\log K_{f^{\mu_{0}(f \nu)-1}} \mid \mu \in\left[\mu_{0}\right], \nu \in\left[\nu_{0}\right]\right\},
$$

where $K_{f^{\mu_{0}\left(f^{\nu}\right)-1}}$ stands for the maximal dilatation of $f^{\mu} \circ\left(f^{\nu}\right)^{-1}$. There always exist quasiconformal mappings which attain the infimum. The Teichmüller distance coincides with the Kobayashi hyperbolic distance (see Gardinar 44 Chapter 7]).

A Beltrami differential $\mu \in B_{1}(R)$ is said to be extremal provided that the 
supremum norm is minimal in its equivalence class. It is known that a Beltrami differential $\mu$ is extremal if and only if there exists a sequence $\left\{\phi_{j}\right\}_{j=1}^{\infty}$ of elements in $A(R)$ with unit integral norm such that $\|\mu\|_{\infty}=\lim _{j \rightarrow \infty} \int_{R} \mu \phi_{j}$. Such a sequence is called a Hamilton sequence for $\mu$. Note that $\left\{\phi_{j}\right\}$ may not contain a subsequence which converges in norm. A Hamilton sequence $\left\{\phi_{j}\right\}$ is said to be degenerate if it converges to 0 uniformly on compact subsets in $R$.

A Beltrami differential of the form $z \bar{\phi} /|\phi|$ on $R$ with some $z \in \Delta=\{z \in$ $\mathbf{C} ;|z|<1\}$ and $\phi \in A(R)$ is called a Teichmüller differential. Obviously, Teich müller differentials are extremal. It is known that if an equivalence class $[\mu] \in$ $T(R)$ contains a Teichmüller differential, then the Teichmüller differential is the unique extremal differential in the equivalence class $[\mu]$

If $\operatorname{dim} T(R)<\infty$ then each equivalence class $[\mu] \in T(R)$ contains a unique Teichmüller differential. On the other hand, whenever $\operatorname{dim} T(R)=\infty$, there exists an extremal differential with a degenerate Hamilton sequence which is not a Teichmüller differential. Such a differential is constructed, for example, as follows. (This may be a known fact. We represent, however, since it seems hard to find a reference in which this fact is written in the following form.)

Proposition 2.1. If $\operatorname{dim} T(R)=\infty$, there exists an extremal differential on $R$ with a degenerate Hamilton sequence which is not a Teichmüller differential.

Proof. When $\operatorname{dim} T(R)=\infty$, the Banach space $A(R)$ is also infinite dimensional. Hence there exists a sequence $\left\{\phi_{j}\right\}_{j=1}^{\infty} \subset A(R)$ with $\left\|\phi_{j}\right\|=\int_{R}\left|\phi_{j}\right|=1$ for $j=1,2,3 \ldots$, which converges to a quadratic differential $\phi \in A(R)$ but does not converges in norm. We may assume in addition that $\phi \equiv 0$ since otherwise we may replace $\left\{\phi_{j}\right\}$ by $\left\{\left(\phi_{j}-\phi\right) /\left\|\phi_{j}-\phi\right\|\right\}$. It is easy to see that there exists an exhaustion of $R$ by compact sets $\left\{K_{j}\right\}_{j=1}^{\infty}$ and a subsequence $\left\{\phi_{N_{j}}\right\}_{j=1}^{\infty}$ of $\left\{\phi_{j}\right\}_{j=1}^{\infty}$ such that $\int_{K j \backslash K_{j-1}}\left|\phi_{N_{j}}\right|>1-1 / 2^{j}$ and $\int_{K_{j}}\left|\phi_{N_{k}}\right|<1 / 2^{j}$ for each positive integer $j$ and $k$ with $j<k$. Put

$$
\mu=\overline{\phi_{N_{j}}} /\left|\phi_{N_{j}}\right| \quad \text { on } K_{j} \backslash K_{j-1}, \quad j=1,2,3, \ldots .
$$

Then for each $z \in \Delta$, the Beltrami differential $z \mu$ is an extremal differential with the degenerate Hamilton sequence $\left\{\phi_{N_{j}}\right\}$.

Let $f: \Delta \rightarrow T(R)$ be a holomorphic isometry with respect to the Poincare distance on $\Delta$ and the Teichmüller distance. We shall call the image $f(\Delta)$ a geodesic disc. If $g: \Delta \rightarrow B_{1}(R)$ is a holomorphic mapping such that $g(0)=0$ and that for each $z \in \Delta$ the Beltrami differential $g(z)$ is an extremal differential with $\|g(z)\|_{\infty}=|z|$, then $\pi \circ g(\Delta)$ is a geodesic disc through [0], where $\pi: B_{1}(R) \rightarrow$ 
$T(R)$ is the canonical projection. If a Beltrami differential $\mu \in B_{1}(R)$ is extremal then $\left\{z \mu /\|\mu\|_{\infty} ; z \in \Delta\right\}$ is a geodesic disc through [0] and [ $\left.\mu\right]$.

When $\operatorname{dim} T(R)<\infty$ every geodesic disc through [0] is of the form $\{z \bar{\phi} /$ $|\phi| ; z \in \Delta\}$ for an element $\phi \in A(R)$. Such a geodesic disc is called a Teichmüller disc. When $\operatorname{dim} T(R)=\infty$ there exists a geodesic disc which is not a Teichmüller disc.

Let $M$ be a finite or infinite dimensional complex space, and let $f: \Delta \rightarrow M$ be a holomorphic mapping. We shall say $f$ is rigid if every holomorphic mapping $\tilde{f}: \Delta \times \Delta \rightarrow M$ with $\tilde{f}(\cdot, 0) \equiv f(\cdot)$ on $\Delta$ depends only on the first variable, namely, $\tilde{f}(\cdot, \zeta) \equiv f(\cdot)$ for each $\zeta \in \Delta$.

\section{Holomorphic family of geodesic dises}

In this section, we shall construct holomorphic families of geodesic discs through certain pairs of points in infinite dimensional Teichmüller spaces.

In what follows, the Riemann surface $R$ is assumed to be analytically infinite unless noticed otherwise. In this case, the Fuchsian group $\Gamma$ representing $R$ is either infinitely generated or of the second kind. Let $U \subset R$ be a Jordan domain with analytic boundary. The following fact plays an important role. (For an element of $B_{1}(R)$, triviality is considered with respect to the equivalence relation for $B_{1}(R)$.)

Theorem 3.1 (Taniguchi [16], Maitani [10]). Let $R$ and $U$ be as above. Let $f^{\nu}$ be a quasiconformal self-mapping of $R$ with trivial Beltrami differential $\nu \in B_{1}(R)$ supported in $\bar{U}$. Then the restriction of $f^{\nu}$ to $R \backslash U$ coincides with the identity mapping.

Recall that in the previous section we defined a quasiconformal mapping with a Beltrami differential so that the ambiguity of compositions of conformal mappings is cancelled.

Proof. Taniguchi [16] showed the above statement for a certain class of Riemann surfaces with no border ([16 Theorem 3]). Maitani [10] extended the statement of [16] as follows: if a homology preserving quasiconformal self-mapping $f$ of a Riemann surface is conformal on an end $G$ with infinitely generated homology group, then the restriction of $f$ to $G$ is the identity map. These results covers the statement of the above theorem except for the case that the Fuchsian group $\Gamma$ is finitely generated of the second kind. In this case, the above statement follows immediately from the definition of $f^{\nu}$. 
In general, for a trivial Beltrami differential $\nu$ and a positive number $t \in$ $(0,1), t \nu$ is not necessarily trivial (Gehring [5]). We recall the following example by Reich (see Kra [8] p.250) constructed in the case of the universal Teichmüller space $T(\Delta)$. Set $\tau_{\alpha}(\zeta)=-\alpha \zeta /(1-\alpha \bar{\zeta})$ for $\zeta \in \Delta$ and $\alpha \in \Delta_{1 / 2}=\{\alpha \in \mathbf{C}$; $|\alpha|<1 / 2\}$. If $\alpha \neq 0$, then $\tau_{\alpha}$ is trivial as an element of $B_{1}(\Delta)$ but there exists a positive number $t \in(0,1)$ such that $t \tau_{\alpha}$ is not trivial. Note that the assignment $\alpha \mapsto \tau_{\alpha}$ is a holomorphic mapping of $\Delta_{1 / 2}$ into $B_{1}(\Delta)$. The above theorem enables us to get such a family of Beltrami differentials on $R$.

PRoposition 3.2. There exists a family $\left\{\nu_{\alpha}\right\}_{a \in \Delta_{1 / 2}}$ of Beltrami differentials on $R$ supported in $\bar{U}$, depending holomorphically on the parameter $\alpha$, such that each $\nu_{\alpha}$ is trivial and that $t_{\alpha} \nu_{\alpha}$ is not trivial for some $t_{\alpha} \in(0,1)$.

Proof. Let $B_{1}(\bar{U}, R)$ denote the subset of $B_{1}(R)$ consisting of all Beltrami differentials supported in $\bar{U}$. Let $h: U \rightarrow \Delta$ be a Riemann mapping. Then the assignment $\mu \mapsto h^{*} \mu$ for $\mu \in B_{1}(\Delta)$ is a norm-preserving holomorphic mapping of $B_{1}(\Delta)$ onto $B_{1}(\bar{U}, R)$. Furthermore, if we denote by $T_{\bar{U}}(R)$ the subset of all points in $T(R)$ determined by Beltrami differentials in $B_{1}(\bar{U})$, then the assignment $\mu \mapsto h^{*} \mu$ induces a bijection $H: T(\Delta) \rightarrow T_{\bar{U}}(R)$ by [16 Proposition 2] and Theorem 3.1. Hence $\mu \in B_{1}(\Delta)$ is trivial with respect to the equivalence relation in $B_{1}(\Delta)$ if and only if $h^{*} \mu \in B_{1}(\bar{U}, R)$ is trivial with respect to the equivalence relation in $B_{1}(R)$. Now for the Reich's family $\left\{\tau_{\alpha}\right\}_{\alpha \in \Delta_{1 / 2}}$, which is recalled above, put $\nu_{\alpha}=h^{*} \tau_{\alpha}$. It follows that the family $\left\{\nu_{\alpha}\right\}_{\alpha \in \Delta_{1 / 2}}$ has the desired property.

Note that for each $\alpha \in \Delta_{1 / 2}$ the set of positive numbers $t$ for which $t \nu_{\alpha}$ is trivial is discrete, since the mapping $z \mapsto\left[z \nu_{\alpha}\right]$ is a non-constant holomorphic mapping of $\Delta$ into $T(R)$.

Theorem 3.1 also implies the following facts.

Lemma 3.3. Let $\nu \in B_{1}(R)$ be a trivial Beltrami differential supported in $\bar{U}$, and $\mu \in B_{1}(R)$ be a Beltrami differential supported in $R \backslash U$. Then

$$
f^{\mu+\nu}=f^{\mu} \circ f^{\nu}
$$

in particular $[\mu+\nu]=[\mu]$.

Proof. Let $\delta$ denote the Beltrami differential of $f^{\mu} \circ f^{\nu}$. Then for each local parameter $z$ around a point $p \in R$, 


$$
\delta=\frac{\mu \circ f^{\nu} \times \overline{f_{z}^{\nu}} / f_{z}^{\nu}+\nu}{1+\mu \circ f^{\nu} \times \bar{\nu} \times \overline{f_{z}^{\nu}} / f_{z}^{\nu}} .
$$

Since $\nu$ is trivial and supported in $\bar{U}$, it follows from Theorem 3.1 that $f^{\nu}(U)$ $=U$ and $f^{\nu} \mid R \backslash U \equiv$ id. Hence if $p \in U$ then $f^{\nu}(p) \in U, \mu \circ f^{\nu}(p)=0$. Therefore

$$
\delta=\frac{0+\nu}{1+0}=\nu \quad \text { on } U
$$

If $p \in R \backslash U$, then $f^{\nu}(z)=z$ and $\nu(p)=0$. Hence we have

$$
\delta=\frac{\mu \times 1+0}{1+0}=\mu \quad \text { on } R \backslash U .
$$

We conclude from the above two formulae that $\delta=\mu+\nu$.

Lemma 3.4. Let $\nu \in B_{1}(R)$ be supported in $\bar{U}$, and $\mu \in B_{1}(R)$ be supported in $R \backslash U$. If $\nu$ is not trivial, then $[\mu+\nu] \neq[\mu]$.

Proof. Assume that $\nu$ is not trivial and that $[\mu+\nu]=[\mu]$. We shall draw a contradiction.

First, note that $f^{\mu+\nu}(U)=f^{\mu}(U)$ (recall that $f^{\mu+\nu}(R)=f^{\mu}(R)$ under the assumption $[\mu+\nu]=[\mu])$. In fact $f^{\mu+\nu} \circ\left(f^{\mu}\right)^{-1}$ is a trivial quasiconformal self-mapping of the Riemann surface $R^{\mu}=f^{\mu}(R)$ and that its Beltrami differential is supported in $f^{\mu}(\bar{U})$. Hence, by Theorem 3.1, $f^{\mu+\nu}(U)=f^{\mu}(U)$ and $f^{\mu+\nu}$ 。 $\left(f^{\mu}\right)^{-1} \mid R \backslash U \equiv \mathrm{id}$.

From the above remark it follows that $\left(f^{\mu}\right)^{-1} \circ f^{\mu+\nu}$ is a trivial quasiconformal mapping with Beltrami differential supported in $\bar{U}$. Let $\tau$ denote the Beltrami differential of $\left(f^{\mu}\right)^{-1} \circ f^{\mu+\nu}$. Then by definition $f^{\mu} \circ f^{\tau}=f^{\mu+\nu}$. On the other hand, the Beltrami differential of $f^{\mu} \circ f^{\tau}$ is $\mu+\tau$ by Lemma 3.3. It follows that $\nu=\tau$. This is a contradiction since $\tau$ is trivial while $\nu$ is not.

Now, we note that there exists extremal Beltrami differntials which vanish on $U$, whenever $R$ is not analytically finite. In fact, as is noted in the previous section, there exists an extremal Beltrami differential $\mu_{0}$ on $R$ with a degenerate Hamilton sequence whenever $R$ is not analytically finite. For such a Beltrami differntial $\mu_{0}$, set

$$
\begin{aligned}
\mu & =\mu_{0} & & \text { on } R \backslash U \\
& =0 & & \text { on } U .
\end{aligned}
$$

Then $\mu$ is an extremal Beltrami differential with the same degenerate Hamilton 
sequence as that for $\mu_{0}$.

THEOREM 3.5. Let $\mu$ be an extremal Beltrami differential which vanishes on $U$ and does not vanish identically on $R$. Then there exists a family of geodesic discs through $[0]$ and $[\mu]$ with a complex analytic parameter. In particular, geodesic lines through certain pairs of points in $T(R)$ are not unique whenever $\operatorname{dim} T(R)=\infty$

Proof. Let $\left\{\nu_{\alpha}\right\}_{\alpha \in \Delta_{1 / 2}}$ is the family of trivial Beltrami differentials supported in $U$ as constructed in Proposition 3.2. Put $\Delta_{r}=\{z \in \Delta ;|z|<r\}$ where $r=$ $\|\mu\|_{\infty} /\left(1+\|\mu\|_{\infty}\right)$. We define a holomorphic mapping $\Phi: \Delta \times \Delta_{r} \rightarrow T(R)$ by

$$
\Phi(z, \alpha)=\left[z \frac{\mu+\nu_{\alpha}}{\|\mu\|_{\infty}}\right],
$$

where $(z, \alpha) \in \Delta \times \Delta_{r}$. Note that $\|\mu\|_{\infty}>\left\|\nu_{\alpha}\right\|_{\infty}$ for $\alpha \in \Delta_{r}$. Hence for each $(z, \alpha) \in \Delta \times \Delta_{r}$ the Beltrami differential $z\left(\mu+\nu_{\alpha}\right) /\|\mu\|_{\infty}$ is extremal with the same degenerate Hamilton sequence as that for $\mu$. It follows that for each $\alpha \in \Delta_{r}$ the holomorphic mapping $z \mapsto \Phi(z, \alpha)$ is an isometry of $\Delta$ into $T(R)$ with respect to the Poincare distance in $\Delta$ and the Teichmüller distance.

Now we verify that $\Phi: \Delta \times \Delta_{r} \rightarrow T(R)$ has the desired property. First, for each $\alpha \in \Delta_{r}$, the geodesic disc $\Phi(\Delta, \alpha)$ contains $\Phi(0, \alpha)=[0]$ and $\Phi\left(\|\mu\|_{\infty}, \alpha\right)$ $=\left[\mu+\nu_{\alpha}\right]=[\mu]$. The last equality follows from the assumption of $\nu_{\alpha}$ to be trivial and Lemma 3.3. Next, note that the holomorphic mapping $\Phi$ depends on the second variable $\alpha$ non-trivially, since $t \nu_{\alpha} /\|\mu\|_{\infty}$ is non-trivial for some positive number $t$, we have $\Phi(t, \alpha)=\left[t \mu /\|\mu\|_{\infty}+t \nu_{\alpha} /\|\mu\|_{\infty}\right] \neq\left[t \mu /\|\mu\|_{\infty}\right]=\Phi(t, 0)$ by Lemma 3.4 .

\section{Examples of rigid holomorphic mappings}

As we have shown above, there exist non-rigid holomorphic proper mappings into $T(R)$. Namely, there exists a family of holomorphic proper mappings in the form $(z, \zeta) \mapsto[z \mu+\zeta \nu]$, where $\mu$ and $\nu$ are supported in disjoint sets, whenever $\operatorname{dim} T(R)=\infty$, in contrast with finite dimensional cases.

In this section we shall give a sufficient condition for a holomorphic mapping of $\Delta$ into the infinite dimensional Teichmüller space $T(R)$ to be rigid.

For this purpose we introduce the Bers embedding. Let $Q(\Gamma)$ denote the space of all bounded holomorphic quadratic differentials for $\Gamma$ on the lower half plane $L$ :

$Q(\Gamma)=\left\{\phi ;\right.$ holomorphic on $\left.L, \phi \circ \gamma \cdot\left(\gamma^{\prime}\right)^{2}=\phi \forall \gamma \in \Gamma, \sup _{z \in L}\left|(\operatorname{Im} z)^{2} \phi(z)\right|<\infty\right\}$. 
(Recall $\Gamma$ is a Fuchsian group acting on $\mathbf{H}$ such that $\mathbf{H} / \Gamma=R$.) The space $Q(\Gamma)$ is a Banach space with norm $\|\phi\|=\sup _{z \in L}\left|(\operatorname{Im} z)^{2} \phi(z)\right|$ for $\phi \in Q(\Gamma)$. For each $\mu \in B_{1}(R)$, set

$$
\begin{aligned}
\mu^{\prime} & =p^{*} \mu & & \text { on } \mathbf{H} \\
& =0 & & \text { on } L,
\end{aligned}
$$

where $p: \mathbf{H} \rightarrow R$ is the canonical projection. Then there exists a unique quasiconformal self-mapping $f_{\mu}$ of $\hat{\mathbf{C}}$ with Beltrami differential $\mu^{\prime}$ such that

$$
f_{\mu}(z)=(z+i)^{-1}+o(1) \text {, near } z=-i .
$$

Let $S\left(f_{\mu}\right)$ denote the Schwarzian derivative of $f_{\mu} \mid L$. Then the assignment $\mu \mapsto$ $S\left(f_{\mu}\right)$ is a holomorphic mapping of $B_{1}(R)$ into $Q(\Gamma)$ and induces a holomorphic injection of $T(R)$ onto a bounded domain in $Q(\Gamma)$. This mapping $T(R) \rightarrow Q(\Gamma)$ is called the Bers embedding of $T(R)$ (see Lehto [9 Chapter V]). Let $T(\Gamma)$ denote the image of $T(R)$. We shall identify $T(R)$ with $T(\Gamma)$ and regard a holomorphic mapping into $T(R)$ as a holomorphic mapping into $T(\Gamma) \subset Q(\Gamma)$.

For each $\phi \in Q(\Gamma)$, let $W_{\phi}$ be the uniquely determined locally schlicht meromorphic function on $L$ with Schwarzian derivative $\phi$ with $W_{\phi}(z)=(z+i)^{-1}$ $+o(1)$ near $z=-i$. It is known that $T(L)$ is exactly the set of all points $\phi \in Q(\Gamma)$ such that $W_{\phi}$ can be extended to quasiconformal self-mappings of $\hat{\mathbf{C}}$. Let $S(\Gamma)$ denote the set of all points $\phi \in Q(\Gamma)$ such that $W_{\phi}$ are schlicht on $L$. Than it is easy to see that $\overline{T(\Gamma)} \subset S(\Gamma)$.

When $T(\Gamma)$ is finite dimensional we utilized the Fatou's theorem and Riesz' theorem on bounded analytic functions to show rigidity of holomorphic proper mappings into $T(\Gamma)$ ([17]). Even when $T(\Gamma)$ is infinite dimensional, Fatou-Riesz type claim is shown for holomorphic mappings of $\Delta$ into $T(\Gamma)$. It is not hard to verify this directly from the definition of $T(\Gamma)$. However, more generally, it is known that Fatou-Riesz type claim is valid for certain holomorphic functions in $\Delta$ with values in Banach spaces. For example, let $X$ be a dual space of a separable Banach space $Y$. Then every holomorphic mapping $\Delta \rightarrow X$ has non-tangential limits almost everwhere in $\partial \Delta$ in weak topology, hence in the strong topology by the theorem of Hensgen. See Hensgen [6] for more details and references. Now, note that the space $Q(\Gamma)$ is identified with a closed subspace of the dual space of the Banach space $A_{1}(L / \Gamma)$ of all integrable holomorphic quadratic differentials on the Riemann surface $L / \Gamma$. Hence we have the following.

PROPOSITION 4.1. Each holomorphic mapping $f: \Delta \rightarrow T(\Gamma)$ has radial limits in $\overline{T(\Gamma)}$ almost everywhere in $\partial \Delta$. If two holomorphic mappings $f: \Delta \rightarrow T(\Gamma)$ and 
$g: \Delta \rightarrow T(\Gamma)$ has the same radial limits on a positive measure set in $\partial \Delta$ then $f \equiv g$ on $\Delta$.

Now, we present a sufficient condition for a mapping of $\Delta$ into the infinite dimensional domain $T(\Gamma)$ to be rigid.

Proposition 4.2. Let $f: \Delta \rightarrow T(\Gamma)$ be a holomorphic mapping. Assume that there exists a positive measure set $E \subset \partial \Delta$ such that for each $e^{i \theta} \in E$ the area of the set $\widehat{\mathbf{C}} \backslash W_{f *\left(e^{i \theta}\right)}(L)$ is zero, where $f_{*}\left(e^{i \theta}\right)$ stands for the radial limit of $f$ at $e^{i \theta}$. Then $f$ is rigid.

Before proving this fact we exhibit some examples.

EXAmple 1. Let $f: \Delta \rightarrow T(1)$ be a holomorphic mapping into the universal Teichmüller space defined by $f(\lambda)=[\lambda]$. Then for each $\lambda \in \Delta$, the function $f_{\lambda}(\cdot)$ is a composition of Möbius transformations and the quasiconformal mapping $z \mapsto z$ $+\lambda \bar{z}$ for $z \in \Delta$ and $z \mapsto z+\lambda / z$ for $z \in \widehat{\mathbf{C}} \backslash \Delta$. Hence it is easy to see that $f$ satisfies the assumption of Proposition 4.2. Therefore $f$ is rigid.

EXAmple 2. Let $S$ be an analytically finite Riemann surface and let $G$ be a Fuchsian group acting on $\mathbf{H}$ and $\mathbf{H} / G=S$. There exists a natural inclusion map $i: T(G) \rightarrow T(1)$, which is known to be proper. Let $f: \Delta \rightarrow T(G)$ be a holommorphic proper map. Then almost every radial limit $f_{*}\left(e^{i \theta}\right)$ of $i \circ f: \Delta \rightarrow T(1)$ corresponds to a totally degenerate boundary group (Shiga [15 Theorem 5]), hence the area of $\widehat{\mathbf{C}} \backslash W_{f *\left(e^{i \theta}\right)}(L)$ is zero by Thurston's theorem. It follows that $i \circ f$ is rigid. When $S$ is of $(0,4)$ type or $(1,1)$ type, the fact that the area of $\widehat{\mathbf{C}} \backslash W_{f *\left(e^{i \theta}\right)}(L)$ is zero follows also from Matsuzaki's result [12], who proved the Ahlfors conjecture affirmatively for two generator groups.

LEMMA 4.3. Let $\phi$ be an element of $S(\Gamma)$ such that the area of $\widehat{\mathbf{C}} \backslash W_{\phi}(L)$ is zero. Then every holomorphic mapping $f: \Delta \rightarrow S(\Gamma)$ with $f(0)=\phi$ is constant.

Proof. The proof is parallel to the argument in Shiga [14 Theorem 5]. For a holomorphic mapping $f: \Delta \rightarrow S(\Gamma)$ with $f(0)=\phi$, put $g(\lambda, z)=W_{f(\lambda)} \circ W_{f(0)}^{-1}(z)$ for $(\lambda, z) \in \Delta \times W_{f(0)}(L)$. Then $g$ is an admissible map in the sense of Bers-Royden [3]. Hence for each $\lambda \in \Delta, g(\lambda, \cdot)$ is extended to a quasiconformal homeomorphism of $\widehat{\mathbf{C}}$ with Beltrami differential supported in $\widehat{\mathbf{C}} \backslash W_{\phi}(L)$ By the assumption the area of this set is zero, hence $g(\lambda, \cdot)$ is a Möbius transformation. 
By the behavior of $W_{f(\lambda)}$ and $W_{f(0)}$ near $z=-i$, the Möbius transformation $g(\lambda, \cdot)$ is the identity map. It follows that $W_{f(\lambda)}(\cdot) \equiv W_{f(0)}(\cdot)$, that is, $f(\lambda)$ $=f(0)$.

Proof of Proposition 4.2. Let $f: \Delta \rightarrow T(\Gamma)$ be a holomorphic mapping, and assume that there exists a positive measure set $E \subset \partial \Delta$ such that for each $e^{\mathrm{i} \theta} \in E$ the area of the set $\widehat{\mathbf{C}} \backslash W_{f *\left(e^{i \theta}\right)}(L)$ is zero. Let $\tilde{f}: \Delta \times \Delta \rightarrow T(\Gamma)$ be a holomorphic mapping with $\tilde{f}(\cdot, 0)=f(\cdot)$. For each $e^{i \theta} \in E$ and any sequence $\left\{z_{m}\right\}$ converging to $e^{i \theta}$, an argument similar to Proposition 4.1 guarantees a subsequence $\left\{z_{m}\right\} \subset$ $\left\{z_{m}\right\}$ such that $\tilde{f}\left(z_{m}, \cdot\right): \Delta \rightarrow T(\Gamma)$ converge to a holomorphic mapping $\tilde{f}_{*}\left(e^{i \theta}, \cdot\right)$ : $\Delta \rightarrow S(\Gamma)$, and that the convergence is uniform in compact subsets in $\Delta$. Hence $\tilde{f}_{*}\left(e^{i \theta}, \cdot\right): \Delta \rightarrow S(\Gamma)$ is a holomorphic mapping such that $\tilde{f}_{*}\left(e^{i \theta}, 0\right)=\tilde{f}_{*}\left(e^{i \theta}, \cdot\right)$. By Lemma 4.3 , the holomorphic mapping $\tilde{f}_{*}\left(e^{i \theta}, \cdot\right)$ is constant. It follows that holomorphic mappings $\tilde{f}(\cdot, 0)$ and $\tilde{f}(\cdot, \zeta)$ have the same radial limit at every $e^{i \theta} \in E$ for each $\zeta \in \Delta$. By Proposition 4.1, $\tilde{f}(\cdot, \zeta) \equiv \tilde{f}(\cdot, 0)$ for each $\zeta$ $\in \Delta$. It follows that $f$ is rigid.

For a finitely generated Fuchsian group of the first kind $G$, it is known that each $\phi \in S(G) \backslash T(G)$ either corresponds to a cusp or $W_{\phi}(L)$ is dense in $\widehat{\mathbf{C}}$ (Bers [2], Maskit [11]). However, the argument as above yields the following.

Proposition 4.4. For any infinitely generated Fuchsian group $\Gamma$ of the first kind there exists analytic sets in $\partial T(\Gamma)$ consisting of non-cusps such that for no point $\phi$ in it $W_{\phi}(L)$ is dense in $\widehat{\mathbf{C}}$

Proof. Let $U$ be a regular domain in $R=\mathbf{H} / \Gamma$ which contains at least two homotopically independent Jordan curves in $R$, and let $\mu$ be an extremal Beltrami differential which vanishes on $U$. Set $f(z)=\left[z \mu /\|\mu\|_{\infty}\right]$ for $z \in \Delta$. Then the holomorphic mapping $f$ is proper, hence very radial limit is contained in $\partial T(\Gamma)$. For each $z \in \Delta$, the quasiconformal mapping $f_{z \mu /\|\mu\|}$, defined in the beginning of this section, is conformal in $p^{-1}(U) \subset \mathbf{H}$, where $p: \mathbf{H} \rightarrow R$ is the canonical projection. Hence there exists no radial limit $\phi$ of $f$ such that $W_{\phi}(L)$ is dense in $\widehat{\mathbf{C}}$ by Abikoff [1 Lemma 3]. On the other hand, exactly as in the finite dimensional cases (Shiga [15 Theorem 5], almost every radial limit is non-cusp. Note that the mapping $f$ is non rigid. Let $\tilde{f}: \Delta \times \Delta \rightarrow T(\Gamma)$ be a holomorphic mapping with $\tilde{f}(\cdot, 0) \equiv f(\cdot)$. Then for almost every $e^{i \theta} \in \partial \Delta$ such that the radial limit $f_{*}\left(e^{i \theta}\right)$ has the above property, $\tilde{f}_{*}\left(e^{i \theta}, \cdot\right)$ is a non-constant holomorphic mapping (cf. Rudin [13 Lemma 15.2.3], see also [18 Section 4]). Now the existence of analytic set in $\partial T(\Gamma)$ as in the statement is obvious. 


\section{REFERENCES}

[1] W. Abikoff, On boundaries of Teichmüller spaces and on Kleinian groups III, Acta. Math., 134 (1975), 217-247

[2] L. Bers, On boundaries of Teichmüller spaces and on Kleinian groups I, Ann. of Math., 91 (1970), 570-600.

[3] L. Bers and H. J. Royden, Holomorphic families of injections, Acta. Math., 157 (1986), 159-186.

[4] F. Gardiner, Teichmüller theory and quadratic differentials, John Wiley and Sons, 1987.

[5] F. Gehring, Quasiconformal mappings which hold the real axis pointwise fixed, Mathematical essays dedicated to A. J. Macintyre, Ohio Univ Press, 1970, pp. $145-158$.

[6] W. Hensgen, Some remarks on boundary values of vector-valued harmonic and analytic functions, Arch. Math., 57 (1991), 88-96.

[7] S. Kobayashi, Hyperbolic Manifolds and Holomorphic Mappings, Dekker, 1970.

[8] I. Kra, On Nielsen-Thurston-Bers type of some self-maps of Riemann surfaces, Acta Math., 146 (1981), 231-270

[9] O. Lehto, Univalent functions and Teichmüller spaces, Springer-Verlag, 1985.

[10] F. Maitani, On the rigidity of an end under conformal mappings preserving the infinite homology bases, (preprint).

[11] B. Maskit, On boundaries of Teichmüller spaces and on Kleinian groups II, Ann. of Math., 91 (1970), 607-639.

[12] K. Matsuzaki, A characterization of extended Schottky type groups with a remark to Ahlfors' conjecture, J. Math. Kyoto Univ., 31 (1991), 259-264.

[13] W. Rudin, Function theory in the unit ball of $C^{n}$, Springer Verlag, 1980.

[14] H. Shiga, On analytic and geometrties of Teichmüller spaces, J. Math. Kyoto Univ., 24 (1984), 441-452.

[15] - Characterization of quasidisks and Teichmüller spaces, Tôhoku Math. J., 37 (1985), 541-552.

[16] M. Taniguchi, On the rigidity of an infinite Riemann surface, Complex Variables, 14 (1990), $161-167$.

[17] H. Tanigawa, Holomorphic mappings into Teichmüller spaces, (to appear in Proc. A. M. S.).

[18] - Rigidity and boundary behavior of holomorphic mappings, (to appear in J. Math. Soc. Japan).

[19] W. Thurston, The geometry and topology of 3-manifold, Lecture Notes, Princeton Univ., 1978-1979.

[20] L. Zhong, Non-uniqueness of geodesics in infinite dimensional Teichmüller spaces, Complex Variables, 16 (1991), 261-272.

Department of Mathematics

Nagoya University

Nagoya 464-01, Japan 